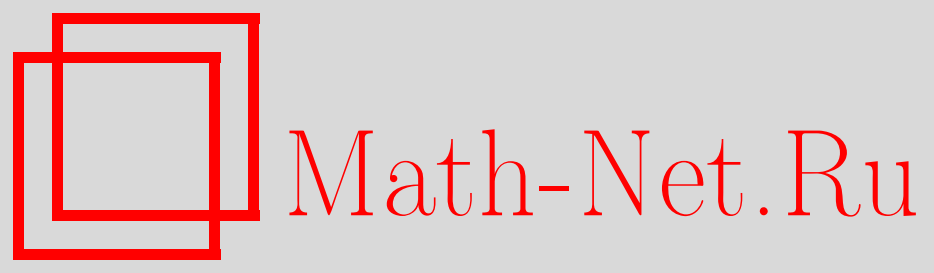

А. Г. Сергеев, Гипотеза о гармонических сферах, ТMФ, 2010, том 164, номер 3, 368-379

DOI: https://doi.org/10.4213/tmf6546

Использование Общероссийского математического портала Math-Net.Ru подразумевает, что вы прочитали и согласны с пользовательским соглашением http: //www . mathnet.ru/rus/agreement

Параметры загрузки:

IP : 3.89 .185 .249

26 апреля 2023 г., 12:51:48

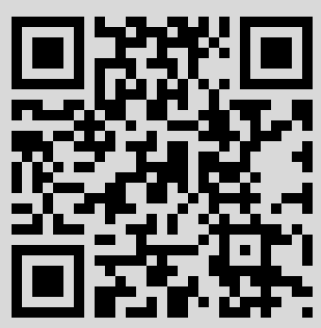




\section{ГИПОТЕЗА О ГАРМОНИЧЕСКИХ СФЕРАХ}

Обсуждается гипотеза о гармонических сферах, связывающая пространство гармонических отображений римановой сферы в пространство петель компактной группы Ли $G$ с пространством модулей $G$-полей Янга-Миллса на четырехмерном евклидовом пространстве.

Ключевые слова: гармоническое отображение, поле Янга-Миллса, пространство петель, твисторное пространство.

\section{1. ВВЕДЕНИЕ}

Известная теорема Атьи [1] устанавливает взаимно однозначное соответствие между пространством модулей $G$-инстантонов на четырехмерном евклидовом пространстве $\mathbb{R}^{4}$ и пространством центрированных голоморфных отображений римановой сферы $\mathbb{P}^{1}$ в пространство петель $\Omega G$ компактной группы Ли $G$. Гипотеза о гармонических сферах получается из этой теоремы "овеществлением": она утверждает, что должно существовать естественное взаимно однозначное соответствие между пространством модулей $G$-полей Янга-Миллса на $\mathbb{R}^{4}$ и пространством центрированных гармонических отображений римановой сферы $\mathbb{P}^{1}$ в пространство петель $\Omega G$.

Доказательство теоремы Атьи опирается на теорему Дональдсона [2], устанавливающую взаимно однозначное соответствие между пространством модулей $G$-инстантонов на $\mathbb{R}^{4}$ и множеством классов эквивалентности голоморфных $G^{\mathbb{C}}$-расслоений над $\mathbb{P}^{1} \times \mathbb{P}^{1}$, голоморфно тривиальных на объединении $\mathbb{P}_{\infty}^{1} \cup \mathbb{P}_{\infty}^{1}$ "бесконечно удаленных" проективных прямых. Теорему Дональдсона можно рассматривать как двумерную редукцию известной теоремы Атьи-Уорда, устанавливающей соответствие между инстантонами на $\mathbb{R}^{4}$ и голоморфными расслоениями на трехмерном проективном пространстве $\mathbb{P}^{3}$, тривиальными на слоях твисторного расслоения $\mathbb{P}^{3} \backslash \mathbb{P}_{\infty}^{1} \rightarrow \mathbb{R}^{4}$. Доказательство теоремы Дональдсона опирается на метод монад, используемый для построения голоморфных векторных расслоений на проективном пространстве, и тем самым является "чисто комплексным". Поэтому для доказательства гипотезы о гармонических сферах необходимо найти "вещественный" аналог теоремы Дональдсона, иначе говоря, получить аналог этой теоремы для гармонических расслоений.

* Математический институт им. В. А. Стеклова РАН, Москва, Россия. E-mail: sergeev@mi.ras.ru 
Настоящая статья посвящена формулировке гипотезы о гармонических сферах и обсуждению возможных путей ее доказательства.

\section{2. ИНСТАНТОНЫ И ПОЛЯ ЯНГА-МИЛЛСА}

Пусть $G$ - компактная группа Ли и $A$ есть $G$-связность (калибровочный потенииал) на $\mathbb{R}^{4}$, задаваемая 1-формой:

$$
A=\sum_{\mu=1}^{4} A_{\mu}(x) d x_{\mu}
$$

с гладкими коэффициентами $A_{\mu}(x)$, принимающими значения в алгебре Ли $\mathfrak{g}$ группы $G$. Обозначим через $F_{A}$ кривизну связности $A$ (калибровочное поле), задаваемую 2-формой

$$
F_{A}=\sum_{\mu, \nu=1}^{4} F_{\mu \nu}(x) d x_{\mu} \wedge d x_{\nu}
$$

с коэффициентами, вычисляемыми по формуле

$$
F_{\mu \nu}=\partial_{\mu} A_{\nu}-\partial_{\nu} A_{\mu}+\left[A_{\mu}, A_{\nu}\right]
$$

где $\partial_{\mu}:=\partial / \partial x_{\mu}, \mu=1,2,3,4, \mathbf{a}[\cdot, \cdot]$ обозначает коммутатор в алгебре Ли $\mathfrak{g}$.

Определим функционал действия Янга-Миллса формулой

$$
S(A)=\frac{1}{2} \int_{\mathbb{R}^{4}} \operatorname{tr}\left(F_{A} \wedge * F_{A}\right),
$$

где $*$ - оператор Ходжа на $\mathbb{R}^{4}$, а след $\operatorname{tr}$ вычисляется с помощью фиксированного инвариантного скалярного произведения на алгебре Ли $\mathfrak{g}$. Функционал $S(A)$ инвариантен относительно калибровочных преобразований вида

$$
A \rightarrow A_{g}:=g^{-1} d g+g^{-1} A g,
$$

где $g: \mathbb{R}^{4} \rightarrow G$ - гладкое отображение, а элементы группы $G$ действуют на алгебре Ли $\mathfrak{g}$ посредством присоединенного представления. Из инвариантности действия $S(A)$ относительно калибровочных преобразований следует, что функционал $S(A)$ зависит на самом деле не от связности $A$, а только от ее класса по модулю калибровочных преобразований.

ОПРЕДЕЛЕниЕ 1. Калибровочные поля, являющиеся экстремальными для функционала $S(A)$, с конечным действием $S(A)<\infty$ называются полями Янга-Миллса.

Поля Янга-Миллса обладают целочисленным топологическим инвариантом, называемым топологическим зарядом, который вычисляется по формуле

$$
k(A)=\frac{1}{8 \pi^{2}} \int_{\mathbb{R}^{4}} \operatorname{tr}\left(F_{A} \wedge F_{A}\right) .
$$

Если записать форму $F_{A}$ как

$$
F_{A}=F_{+}+F_{-},
$$


где $F_{ \pm}=\left(* F_{A} \pm F_{A}\right) / 2$, то формулы для действия и заряда можно будет переписать в виде

$$
\begin{aligned}
& S(A)=\frac{1}{2} \int_{\mathbb{R}^{4}}\left(\left\|F_{+}\right\|^{2}+\left\|F_{-}\right\|^{2}\right) d^{4} x, \\
& k(A)=\frac{1}{8 \pi^{2}} \int_{\mathbb{R}^{4}}\left(-\left\|F_{+}\right\|^{2}+\left\|F_{-}\right\|^{2}\right) d^{4} x,
\end{aligned}
$$

где норма $\|\cdot\|^{2}$ вычисляется с помощью инвариантного скалярного произведения на алгебре Ли g. Сравнивая эти формулы, немедленно получаем, что

$$
S(A) \geqslant 4 \pi^{2}|k(A)|,
$$

причем равенство в последнем неравенстве может достигаться при $k>0$ только на решениях уравнения

$$
* F_{A}=-F_{A},
$$

а при $k<0$ - только на решениях уравнения

$$
* F_{A}=F_{A} .
$$

ОПредЕЛЕНиЕ 2. Решения с конечным действием $S(A)<\infty$ уравнения (1) называются инстантонами, а решения уравнения (2) - антиинстантонами.

(Анти)инстантоны реализуют локальные минимумы действия $S(A)$, однако помимо них имеются и другие, неминимальные, критические точки этого функционала.

\section{3. ГАРМОНИЧЕСКИЕ СФЕРЫ}

Начнем с модельного примера: рассмотрим гладкое отображение $\varphi: \mathbb{R}^{2} \rightarrow S^{2}$ евклидовой плоскости $\mathbb{R}^{2}$ в двумерную сферу $S^{2} \subset \mathbb{R}^{3}$. Определим энергию $\varphi$ интегралом Дирихле

$$
E(\varphi)=\frac{1}{2} \int_{\mathbb{R}^{2}}|d \varphi|^{2} d x_{1} d x_{2}
$$

и будем искать отображения $\varphi: \mathbb{R}^{2} \rightarrow S^{2}$ с конечной энергией $E(\varphi)<\infty$, являющиеся экстремальными по отношению к функционалу $E(\varphi)$. Из условия конечности энергии вытекает, что искомые отображения $\varphi$ должны стабилизироваться на бесконечности, т. е. $\varphi(x) \rightarrow x_{0}$ при $|x| \rightarrow \infty$. Поэтому они продолжаются на компактификацию $S^{2}$ плоскости $\mathbb{R}^{2}$ до непрерывных отображений

$$
\varphi: S^{2}=\left(\mathbb{R}^{2} \cup \infty\right) \rightarrow S^{2} \subset \mathbb{R}^{3} .
$$

Такие отображения обладают топологическим инвариантом - степенъю отображения, вычисляемой по формуле

$$
\operatorname{deg} \varphi:=\int_{\mathbb{R}^{2}} \varphi^{*} \omega
$$

где $\omega$ - нормированная форма объема на сфере $S^{2}$. 
Введем комплексную координату $z=x_{1}+i x_{2}$ в области определения $\mathbb{R}^{2}$ отображения $\varphi$ и стереографическую комплексную координату $w$ в области его значений $S^{2} \subset \mathbb{R}^{3}$. Тогда формулы для энергии и степени отображения перепишутся в этих координатах в виде

$$
\begin{aligned}
E(\varphi) & =2 \int_{\mathbb{C}} \frac{\left|w_{z}\right|^{2}+\left|w_{\bar{z}}\right|^{2}}{\left(1+|w|^{2}\right)^{2}}|d z \wedge d \bar{z}|, \\
\operatorname{deg} \varphi & =\frac{1}{2 \pi} \int_{\mathbb{C}} \frac{\left|w_{z}\right|^{2}-\left|w_{\bar{z}}\right|^{2}}{\left(1+|w|^{2}\right)^{2}}|d z \wedge d \bar{z}| .
\end{aligned}
$$

Сравнивая эти формулы, немедленно получаем, что

$$
E(\varphi) \geqslant 4 \pi|\operatorname{deg} \varphi|
$$

причем равенство может достигаться при $\operatorname{deg} \varphi \geqslant 0$ только на голоморфных функциях $w=\varphi(z)$, а при $\operatorname{deg} \varphi<0$ - только на антиголоморфных функциях $w=\varphi(z)$. Можно показать, что других критических точек у функционала $E(\varphi)$ нет.

Теперь нетрудно описать все локальные минимумы функционала $E(\varphi)$. Ввиду $S O(3)$-инвариантности задачи можно положить $\varphi_{0}=1$. Считая степень $\operatorname{deg} \varphi$ положительной, получаем, что все минимумы функционала $E(\varphi)$ с фиксированной степенью $k=\operatorname{deg} \varphi$ задаются рациональными функциями вида

$$
w=\varphi(z)=\prod_{j=1}^{k} \frac{z-a_{j}}{z-b_{j}},
$$

где $a_{j} \neq b_{j}-$ произвольные комплексные числа.

Будем называть отображения $\varphi: \mathbb{R}^{2} \rightarrow S^{2}$, являющиеся экстремальными для функционала энергии $E(\varphi)$, гармоническими. Из наших рассуждений вытекает, что все гармонические отображения $\varphi: \mathbb{P}^{1} \rightarrow \mathbb{P}^{1}$ на самом деле задаются либо голоморфными, либо антиголоморфными отображениями.

Обобщая приведенный модельный пример, мы будем рассматривать гладкие отображения $\varphi: \mathbb{P}^{1} \rightarrow N$ из римановой сферы $\mathbb{P}^{1}$ в ориентируемые римановы многообразия $N$.

ОПредЕЛЕНиЕ 3. Гладкое отображение $\varphi: \mathbb{P}^{1} \rightarrow N$ из римановой сферы в ориентируемое риманово многообразие $N$ называется гармоническим, если оно является экстремальным по отношению к функционалу энергии, задаваемому интегралом Дирихле

$$
E(\varphi)=\frac{1}{2} \int_{\mathbb{C}}|d \varphi|_{N}^{2} \frac{|d z \wedge d \bar{z}|}{\left(1+|z|^{2}\right)^{2}},
$$

где модуль дифференциала $d \varphi$ вычисляется в метрике $|\cdot|_{N}$ многообразия $N$.

Если многообразие $N$ кэлерово, т. е. обладает комплексной структурой, совместимой с римановой метрикой, то, как и в разобранном примере, голоморфные и антиголоморфные отображения $\varphi: \mathbb{P}^{1} \rightarrow N$ являются локальными минимумами функционала энергии $E(\varphi)$. Однако при $\operatorname{dim}_{\mathbb{C}} N>1$ имеются, как правило, и не минимальные гармонические отображения. 
Сравнивая гармонические отображения с полями Янга-Миллса, введенными в предыдущем разделе, нельзя не заметить очевидную параллель:

$$
\left\{\begin{array}{l}
(\text { анти }) \text { голоморфные } \\
\text { отображения }
\end{array}\right\} \longleftrightarrow\{(\text { анти }) \text { инстантоны }\}
$$

и

$$
\left\{\begin{array}{l}
\text { гармонические } \\
\text { отображения }
\end{array}\right\} \longleftrightarrow\{\text { поля Янга-Миллса }\} \text {. }
$$

Согласно теореме Атьи и гипотезе о гармонических сферах, о которых речь пойдет ниже, эта внешняя аналогия имеет гораздо более глубокий смысл.

\section{4. ТВИСТОРНАЯ ИНТЕРПРЕТАЦИЯ ИНСТАНТОНОВ}

Начнем с построения твисторного расслоения над евклидовым пространством $\mathbb{R}^{4}[3]$. Для этого компактифицируем $\mathbb{R}^{4}$ до евклидовой сферы $S^{4}=\mathbb{R}^{4} \cup\{\infty\}$ и отождествим $S^{4}$ с кватернионной проективной прямой $\mathbb{H} \mathbb{P}^{1}$. Точками $\mathbb{H}^{1}$ являются пары $\left[z_{1}+z_{2} j, z_{1}^{\prime}+z_{2}^{\prime} j\right]$ кватернионов, не равных нулю одновременно, которые заданы с точностью до умножения справа на ненулевой кватернион.

Твисторное расслоение

$$
\pi: \mathbb{P}^{3} \stackrel{\mathbb{P}^{1}}{\longrightarrow} \mathbb{H} \mathbb{P}^{1}
$$

задается тавтологической формулой

$$
\left[z_{1}, z_{2}, z_{3}, z_{4}\right] \mapsto\left[z_{1}+z_{2} j, z_{3}+z_{4} j\right]
$$

в которой четверка комплексных чисел $\left[z_{1}, z_{2}, z_{3}, z_{4}\right]$ определена с точностью до умножения на ненулевое комплексное число, а пара кватернионов $\left[z_{1}+z_{2} j, z_{3}+z_{4} j\right]-$ с точностью до умножения на ненулевой кватернион. Слой расслоения $\pi$ совпадает с комплексной проективной прямой $\mathbb{P}^{1}$.

Сужение твисторного расслоения

$$
\pi: \mathbb{P}^{3} \rightarrow S^{4}
$$

на евклидово пространство $\mathbb{R}^{4}=S^{4} \backslash \infty$ задает твисторное расслоение

$$
\pi: \mathbb{P}^{3} \backslash \mathbb{P}_{\infty}^{1} \rightarrow \mathbb{R}^{4}
$$

в котором выброшенная проективная прямая $\mathbb{P}_{\infty}^{1}$ совпадает со слоем расслоения (3) над бесконечностью. Слой расслоения (4) над точкой $x \in \mathbb{R}^{4}$ можно отождествить с пространством комплексных структур на касательном пространстве $T_{x} \mathbb{R}^{4} \cong \mathbb{R}^{4}$, совместимых с метрикой и ориентацией, а гладкие сечения (4) - с почти комплексными структурами на $\mathbb{R}^{4}$ (см. книгу [3]).

В терминах твисторного расслоения $\pi: \mathbb{P}^{3} \backslash \mathbb{P}^{1} \rightarrow \mathbb{R}^{4}$ пространство модулей $G$-инстантонов, т. е. фактор пространства всех $G$-инстантонов на $\mathbb{R}^{4}$ по модулю калибровочных преобразований, допускает следующую интерпретацию, даваемую теоремой Атьи-Уорда:

$$
\left\{\begin{array}{l}
\text { пространство модулей } \\
G \text {-инстантонов на } \mathbb{R}^{4}
\end{array}\right\} \longleftrightarrow\left\{\begin{array}{l}
\text { классы (центрированной) эквивалентности } \\
\text { голоморфных } G^{\mathbb{C}} \text {-расслоений над } \mathbb{P}^{3}, \text { голо- } \\
\text { морфно тривиальных на слоях проекции } \pi
\end{array}\right\} .
$$


Этот результат допускает двумерную редукцию на пространство $\mathbb{P}^{1} \times \mathbb{P}^{1}$, даваемую теоремой Дональдсона:

$$
\left\{\begin{array}{l}
\text { пространство модулей } \\
G \text {-инстантонов на } \mathbb{R}^{4}
\end{array}\right\} \longleftrightarrow\left\{\begin{array}{l}
\text { классы (центрированной) эквивалентности } \\
\text { голоморфных } G^{\mathbb{C}} \text {-расслоений над } \mathbb{P}^{1} \times \mathbb{P}^{1}, \\
\text { голоморфно тривиальных на объединении } \\
\mathbb{P}_{\infty}^{1} \cup \mathbb{P}_{\infty}^{1}
\end{array}\right\} .
$$

\section{5. ТВИСТОРНАЯ ИНТЕРПРЕТАЦИЯ ГАРМОНИЧЕСКИХ СФЕР}

Пользуясь интерпретацией твисторного расслоения $\mathbb{P}^{3} \rightarrow S^{4}$, приведенной в разделе 4 , можно построить твисторное расслоение над произвольным четномерным ориентируемым римановым многообразием $N$. А именно, определим его как расслоение комплексных структур на многообразии $N$, совместимых с метрикой и ориентацией. Более подробно, твисторное расслоение $\pi: Z \rightarrow N$ есть расслоение, ассоциированное с расслоением ортонормированных реперов на $N$, слоем которого в точке $x \in N$ является пространство комплексных структур $J_{x}$ на касательном пространстве $T_{x} N$, совместимых с метрикой и ориентацией. Это пространство можно отождествить с однородным пространством $S O(2 n) / U(n)$, где $2 n$ - размерность многообразия $N$. Твисторное пространство $Z$ обладает, как показано в работе [4], естественной почти комплексной структурой, обозначаемой через $\mathcal{J}^{1}$. Почти комплексная структура $\mathcal{J}^{1}$ интегрируема, если многообразие $N$ конформно плоско.

Однако для описания гармонических сфер в $N$ нам придется использовать другую почти комплексную структуру, определяемую следующим образом. С помощью связности Леви-Чивита можно ввести порождаемую ею связность на твисторном расслоении $\pi: Z \rightarrow N$. Новая почти комплексная структура на $Z$, обозначаемая через $\mathcal{J}^{2}$, полагается равной $\mathcal{J}^{1}$ в горизонтальных направлениях относительно введенной связности и равной $-\mathcal{J}^{1}$ в вертикальных направлениях. Указанная структура была введена в работе [5] и всегда не интегрируема. В ее терминах гармонические сферы в $N$ допускают следующее описание.

Теорема 1 [5]. Проекиии $\varphi=\pi \circ \psi$ отображений $\psi: \mathbb{P}^{1} \rightarrow Z$, голоморфных относительно почти комплексной структуры $\mathcal{J}^{2}$, на многообразие $N$ являются гармоническими отображениями.

Приведенная теорема позволяет строить гармонические сферы в многообразии $N$ из почти голоморфных сфер в твисторном пространстве $Z$. Тем самым исходная "вещественная" задача построения гармонических сфер на римановом многообразии $N$ сводится к "комплексной" задаче построения голоморфных сфер на почти комплексном многообразии $Z$. На первый взгляд кажется, что последняя задача ничуть не проще исходной, особенно если учитывать тот факт, что почти комплексная структура $\mathcal{J}^{2}$ никогда не интегрируема. Известно, что подобная почти комплексная структура может, например, не допускать даже локально никаких непостоянных голоморфных функций. Заметим, однако, что отображения $\psi: \mathbb{P}^{1} \rightarrow Z$, голоморфные относительно почти комплексной структуры $\mathcal{J}^{2}$, являются решениями $\bar{\partial}_{J}$-уравнения на $\mathbb{P}^{1}$, где $J:=\psi^{*}\left(\mathcal{J}^{2}\right)$ - почти комплексная структура на $\mathbb{P}^{1}$, индуцированная отображением $\psi$ (которая интегрируема, как и любая почти комплексная структура на 
римановой поверхности). Иначе говоря, описание голоморфных сфер в пространстве $\left(Z, \mathcal{J}^{2}\right)$ сводится к решению нелинейного уравнения Коши-Римана на римановой сфере относительно комплексной структуры $J$. В частности, такое уравнение обладает большим запасом локальных решений.

Применим указанный твисторный подход к задаче описания гармонических сфер в комплексном грассмановом многообразии $G_{r}\left(\mathbb{C}^{d}\right)$. В этом случае в качестве твисторных расслоений над $G_{r}\left(\mathbb{C}^{d}\right)$ естественно выбирать расслоения комплексных структур на $G_{r}\left(\mathbb{C}^{d}\right)$, инвариантных относительно действия унитарной группы $U(d)$. Такие расслоения совпадают с флаговыми расслоениями над $G_{r}\left(\mathbb{C}^{d}\right)$, к определению которых мы переходим.

ОПРЕДЕЛЕНИЕ 4. Флаговое многообразие $F_{\mathbf{r}}\left(\mathbb{C}^{d}\right)$ в $\mathbb{C}^{d}$ muna $\mathbf{r}=\left(r_{1}, \ldots, r_{n}\right)$ с $d=r_{1}+\cdots+r_{n}$ состоит из флагов $\mathcal{W}=\left(W_{1}, \ldots, W_{n}\right)$, т. е. наборов вложенных комплексных подпространств

$$
W_{1} \subset \cdots \subset W_{n}=\mathbb{C}^{d}
$$

таких, что размерность пространства $V_{1}:=W_{1}$ равна $r_{1}$, а размерности подпространств $V_{i}:=W_{i} \ominus W_{i-1}$ равны $r_{i}$ при $1<i \leqslant n$.

Флаговое многообразие $F_{\mathbf{r}}\left(\mathbb{C}^{d}\right)$ допускает следующее представление в виде однородного пространства унитарной группы $U(d)$ :

$$
F_{\mathbf{r}}\left(\mathbb{C}^{d}\right)=U(d) / U\left(r_{1}\right) \times \cdots \times U\left(r_{n}\right) .
$$

Это компактное кэлерово многообразие, обладающее $U(d)$-инвариантной комплексной структурой, которую мы обозначаем снова через $\mathcal{J}^{1}$.

Для того чтобы построить твисторное флаговое расслоение над грассмановым многообразием $G_{r}\left(\mathbb{C}^{d}\right)$, фиксируем упорядоченное подмножество $\sigma \subset\{1, \ldots, n\}$ так, чтобы $\sum_{i \in \sigma} r_{i}=r$, и определим флаговое расслоение

$$
\pi_{\sigma}: F_{\mathbf{r}}\left(\mathbb{C}^{d}\right) \rightarrow G_{r}\left(\mathbb{C}^{d}\right)
$$

полагая

$$
\pi_{\sigma}: \mathcal{W}=\left(W_{1}, \ldots, W_{n}\right) \rightarrow W:=\bigoplus_{i \in \sigma} V_{i}
$$

Как в начале этого раздела, можно наделить флаговое расслоение $\pi_{\sigma}$ почти комплексной структурой $\mathcal{J}_{\sigma}^{2}$ так, что для него будет выполняться аналог теоремы 1.

Теорема 2 [6]. Флаговое расслоение

$$
\pi_{\sigma}:\left(F_{\mathbf{r}}\left(\mathbb{C}^{d}\right), \mathcal{J}_{\sigma}^{2}\right) \rightarrow G_{r}\left(\mathbb{C}^{d}\right)
$$

наделенное почти комплексной структурой $\mathcal{J}_{\sigma}^{2}$, является твисторным, т.е. проекция $\varphi=\pi_{\sigma} \circ \psi$ любой почти голоморфной сферь $\psi: \mathbb{P}^{1} \rightarrow F_{\mathbf{r}}\left(\mathbb{C}^{d}\right)$ на $G_{r}\left(\mathbb{C}^{d}\right)$ является гармонической сферой $\varphi: \mathbb{P}^{1} \rightarrow G_{r}\left(\mathbb{C}^{d}\right)$ в $G_{r}\left(\mathbb{C}^{d}\right)$. Верно и обратное: любая гармоническая сфера $\varphi: \mathbb{P}^{1} \rightarrow G_{r}\left(\mathbb{C}^{d}\right)$ в $G_{r}\left(\mathbb{C}^{d}\right)$ может быть построена указанным образом с помощъю некоторого флагового расслоения $\pi_{\sigma}: F_{\mathbf{r}}\left(\mathbb{C}^{d}\right) \rightarrow G_{r}\left(\mathbb{C}^{d}\right)$. 


\section{6. ТЕОРЕМА АТЬИ}

Напомним сначала определение пространства петель компактной группы Ли $G$ :

$$
\Omega G:=L G / G,
$$

где $L G=C^{\infty}\left(S^{1}, G\right)$ есть группа гладких петель в группе $G$, а группа $G$ в знаменателе отождествляется с подгруппой постоянных отображений $S^{1} \rightarrow g_{0} \in G$. Пространство $\Omega G$ является кэлеровым многообразием Фреше, которое обладает $L G$-инвариантной комплексной структурой. Указанная структура возникает из представления $\Omega G$ в виде фактора комплексной группы петель $L G^{\mathbb{C}}$ :

$$
\Omega G=L G^{\mathbb{C}} / L_{+} G^{\mathbb{C}},
$$

где $G^{\mathbb{C}}$ - комплексификация группы $G$, а подгруппа $L_{+} G^{\mathbb{C}}$ состоит из петель $\gamma \in L G^{\mathbb{C}}$, гладко продолжающихся до голоморфных отображений единичного круга $\Delta$ в $G^{\mathbb{C}}$.

Переходя к теореме Атьи, напомним интерпретацию пространства модулей $G$-инстантонов, даваемую теоремой Дональдсона (6). Теорема Атьи утверждает, что правую часть этого соответствия можно отождествить с пространством голоморфных сфер в $\Omega G$. Более подробно, имеет место взаимно однозначное соответствие

$$
\left\{\begin{array}{l}
\text { классы (центрированной) эквива- } \\
\text { лентности голоморфных } G^{\mathbb{C}} \text {-рас- } \\
\text { слоений над } \mathbb{P}^{1} \times \mathbb{P}^{1}, \text { голоморф- } \\
\text { но тривиальных на объединении } \\
\mathbb{P}_{\infty}^{1} \cup \mathbb{P}_{\infty}^{1}
\end{array}\right\} \longleftrightarrow\left\{\begin{array}{l}
\text { центрированные голоморф- } \\
\text { ные сферы } f: \mathbb{P}^{1} \rightarrow \Omega G, \text { пе-- } \\
\text { реводящие } \infty \text { в начало } \Omega G
\end{array}\right\} .
$$

Доказательство теоремы Атьи основано на следующей конструкции. Рассмотрим сужение голоморфного $G^{\mathbb{C}}$-расслоения над $\mathbb{P}^{1} \times \mathbb{P}^{1}$ на проективную прямую $\mathbb{P}_{z}^{1}$, параллельную $P_{\infty}^{1}$ и проходящую через точку $\mathbb{P}^{1} \times\{z\}$. Оно задается функцией перехода

$$
\tilde{f}_{z}: S^{1} \rightarrow G^{\mathbb{C}}
$$

в покрытии $\mathbb{P}_{z}^{1}=\bar{\Delta}_{+} \cup \bar{\Delta}_{-}$соответственно нижним и верхним полушариями сферы $\mathbb{P}_{z}^{1}$, причем функция $\tilde{f}_{z}$ голоморфна в окрестности экватора $S^{1}=\bar{\Delta}_{+} \cap \bar{\Delta}_{-}$. Следовательно, $\tilde{f}_{z} \in L G^{\mathbb{C}}$, и мы имеем отображение

$$
f: \mathbb{P}^{1} \ni z \rightarrow \tilde{f}_{z} \in L G^{\mathbb{C}} \rightarrow f(z) \in \Omega G=L G^{\mathbb{C}} / L_{+} G^{\mathbb{C}} .
$$

Это отображение голоморфно и центрировано тогда и только тогда, когда исходное $G^{\mathbb{C}}$-расслоение над $\mathbb{P}^{1} \times \mathbb{P}^{1}$ голоморфно и тривиально на объединении $\mathbb{P}_{\infty}^{1} \cup \mathbb{P}_{\infty}^{1}$.

\section{7. ГИПОТЕЗА О ГАРМОНИЧЕСКИХ СФЕРАХ}

Из теорем Дональдсона и Атьи следует, что имеется взаимно однозначное соответствие

$$
\left\{\begin{array}{l}
\text { пространство модулей } \\
G \text {-инстантонов на } \mathbb{R}^{4}
\end{array}\right\} \longleftrightarrow\left\{\begin{array}{l}
\text { центрированные голоморфные } \\
\text { сферы } f: \mathbb{P}^{1} \rightarrow \Omega G
\end{array}\right\} .
$$

Тем самым устанавливается соответствие между локальными минимумами двух введенных ранее функционалов - функционала действия Янга-Миллса, определенного на калибровочных $G$-полях в $\mathbb{R}^{4}$, и функционала энергии, определенного на гладких сферах в пространстве петель $\Omega G$. Напомним, что локальные минимумы действия Янга-Миллса задаются инстантонами и антиинстантонами на $\mathbb{R}^{4}$, а локальные 
минимумы функционала энергии - голоморфными и антиголоморфными сферами в $\Omega G$. Заменяя локальные минимумы на критические точки указанных функционалов, приходим к гипотезе о гармонических сферах, согласно которой должно существовать взаимно однозначное соответствие

$$
\left\{\begin{array}{l}
\text { пространство модулей } \\
G \text {-полей Янга-Миллса } \\
\text { на } \mathbb{R}^{4}
\end{array}\right\} \longleftrightarrow\left\{\begin{array}{l}
\text { центрированные гармонические } \\
\text { сферы } f: \mathbb{P}^{1} \rightarrow \Omega G
\end{array}\right\} .
$$

Описанный переход от локальных минимумов к критическим точкам естественно называть “овеществлением". Действительно, если заменить гладкие сферы в правой части диаграммы на гладкие функции $f: \mathbb{C} \rightarrow \mathbb{C}$, то указанная процедура будет отвечать переходу от голоморфных и антиголоморфных функций к произвольным гармоническим функциям (представляющимся в виде суммы голоморфных и антиголоморфных функций). В случае гладких сфер в пространстве петель $\Omega G$ этот переход от голоморфных и антиголоморфных сфер к гармоническим становится нетривиальным в силу нелинейности уравнений Эйлера-Лагранжа для функционала энергии.

Как уже отмечалось во введении, прямое обобщение теоремы Атьи-Дональдсона на гармонический случай не проходит, поскольку доказательство теоремы Дональдсона, основанное на методе монад, является чисто голоморфным и не распространяется непосредственно на гармонический случай. Однако можно попытаться свести доказательство гипотезы о гармонических сферах к голоморфному случаю, "поднимая" обе части соответствия из гипотезы на отвечающие им твисторные пространства. K сожалению, у нас нет подходящей твисторной интерпретации полей Янга-Миллса, но для правой части соответствия такая интерпретация имеется и будет представлена в следующем разделе.

\section{8. ТВИСТОРНОЕ РАССЛОЕНИЕ НАД ПРОСТРАНСТВОМ ПЕТЕЛЬ}

Идея конструкции твисторного расслоения над пространством петель $\Omega G$ состоит в том, чтобы сначала вложить $\Omega G$ в некоторый бесконечномерный грассманиан, а затем построить твисторное расслоение над этим грассманианом по аналогии с конечномерным случаем.

Начнем с определения бесконечномерного грассманиана, которым мы будем пользоваться. Пусть $H$ есть комплексное гильбертово пространство, моделью которого может служить пространство $L_{0}^{2}\left(S^{1}, \mathbb{C}\right)$ квадратично-интегрируемых функций на окружности с нулевым средним. Предположим, что $H$ обладает поляризацией, т. е. разложением

$$
H=H_{+} \oplus H_{-}
$$

в прямую ортогональную сумму замкнутых бесконечномерных подпространств. В случае $H=L_{0}^{2}\left(S^{1}, \mathbb{C}\right)$ в качестве таких подпространств можно взять

$$
H_{ \pm}=\left\{\gamma \in H: \gamma=\sum_{ \pm k>0} \gamma_{k} e^{i k \theta}\right\}
$$


ОПРЕДЕЛЕНИЕ 5. Грассманиан Гилъберта-Шмидта $\mathrm{Gr}_{\mathrm{HS}}(H)$ состоит из замкнутых подпространств $W \subset H$ таких, что ортогональная проекция $\pi_{+}: W \rightarrow H_{+}$фредгольмова, а ортогональная проекция $\pi_{-}: W \rightarrow H_{-}$является оператором Гильберта-Шмидта.

Для заданного подпространства $W \in \mathrm{Gr}_{\mathrm{HS}}(H)$ фредгольмов индекс проекции $\pi_{+}: W \rightarrow H_{+}$принято называть виртуальной размерностью подпространства $W$.

Грассманиан Гильберта-Шмидта $\mathrm{Gr}_{\mathrm{Hs}}(H)$ допускает однородное представление:

$$
\operatorname{Gr}_{\mathrm{HS}}(H)=\frac{U_{\mathrm{HS}}(H)}{U\left(H_{+}\right) \times U\left(H_{-}\right)},
$$

где унитарная группа Гильберта-Шмидта $U_{\mathrm{HS}}(H)$ есть множество операторов $A \in U(H)$ таких, что $\pi_{-} \circ A \circ \pi_{+}$есть оператор Гильберта-Шмидта.

Грассманиан $\mathrm{Gr}_{\mathrm{HS}}(H)$ является гильбертовым кэлеровым многообразием, состоящим из счетного числа связных компонент фиксированной виртуальной размерности:

$$
\operatorname{Gr}_{\mathrm{HS}}(H)=\bigcup_{d} G_{d}(H)
$$

где

$$
G_{d}(H)=\left\{W \in \operatorname{Gr}_{\mathrm{HS}}(H): \text { virt.dim } W=d\right\} .
$$

Многообразие виртуальных флагов $F_{\mathbf{r}}^{d}(H)$ определяется по аналогии с конечномерным случаем.

ОПРЕДЕЛЕНИЕ 6. Многообразие виртуалъных флагов $F_{\mathbf{r}}^{d}(H)$ в $H$ muna $\mathbf{r}=$ $\left(r_{1}, \ldots, r_{n}\right)$ с $d=r_{1}+\cdots+r_{n}$ состоит из флагов $\mathcal{W}=\left(W_{1}, \ldots, W_{n}\right)$, т. е. наборов вложенных комплексных подпространств

$$
W_{1} \subset \cdots \subset W_{n} \subset H
$$

таких, что виртуальная размерность пространства $V_{1}:=W_{1}$ равна $r_{1}$, а размерности подпространств $V_{i}:=W_{i} \ominus W_{i-1}$ равны $r_{i}$ при $1<i \leqslant n$.

Для построения твисторного флагового расслоения над грассмановым многообразием $G_{r}(H)$ фиксируем упорядоченное подмножество $\sigma \subset\{1, \ldots, n\}$ так, чтобы $\sum_{i \in \sigma} r_{i}=r$, и определим виртуальное флаговое расслоение

$$
\pi_{\sigma}: F_{\mathbf{r}}^{d}(H) \rightarrow G_{r}(H),
$$

полагая

$$
\pi_{\sigma}: \mathcal{W}=\left(W_{1}, \ldots, W_{n}\right) \rightarrow W:=\bigoplus_{i \in \sigma} V_{i}
$$

Как и в конечномерном случае, можно наделить виртуальное флаговое расслоение $\pi_{\sigma}$ почти комплексной структурой $\mathcal{J}_{\sigma}^{2}$ так, что для него будет выполняться аналог теоремы 2 .

Теорема 3. Виртуальное флаговое расслоение

$$
\pi_{\sigma}:\left(F_{\mathbf{r}}^{d}(H), \mathcal{J}_{\sigma}^{2}\right) \rightarrow G_{r}(H),
$$

наделенное почти комплексной структурой $\mathcal{J}_{\sigma}^{2}$, является твисторным, т.е. проекиия $\varphi=\pi_{\sigma} \circ \psi$ любой почти голоморфной сферы $\psi: \mathbb{P}^{1} \rightarrow F_{\mathbf{r}}^{d}(H)$ на $G_{r}(H)$ является гармонической сферой $\varphi: \mathbb{P}^{1} \rightarrow G_{r}(H)$ в $G_{r}(H)$. 
Есть основания ожидать, что в рассматриваемой ситуации верна и вторая часть теоремы 2 , а именно утверждение, обратное к сформулированному.

Построим теперь изометрическое вложение пространства петель в грассманиан Гильберта-Шмидта. Предположим, что компактная группа Ли $G$ реализована в виде подгруппы унитарной группы $U(N)$ и построим вложение $\Omega G$ в грассманиан $\operatorname{Gr}_{\mathrm{HS}}(H)$, где в качестве гильбертова пространства $H$ берется пространство $L_{0}^{2}\left(S^{1}, \mathbb{C}^{N}\right)$.

Построим сначала вложение группы петель $L G$ в унитарную группу ГильбертаШмидта $U_{\mathrm{HS}}(H)$. Для этого сопоставим петле $\gamma$ из пространства $L G=C^{\infty}\left(S^{1}, G\right) \subset$ $C^{\infty}\left(S^{1}, U(N)\right)$ оператор умножения $M_{\gamma}$ в гильбертовом пространстве $H=L_{0}^{2}\left(S^{1}, \mathbb{C}^{N}\right)$, действующий по формуле

$$
H=L_{0}^{2}\left(S^{1}, \mathbb{C}^{N}\right) \ni h \rightarrow M_{\gamma} h(z):=\gamma(z) h(z), \quad z \in S^{1} .
$$

Иначе говоря, $M_{\gamma} h$ есть вектор-функция из $H=L_{0}^{2}\left(S^{1}, \mathbb{C}^{N}\right)$, получаемая поточечным применением матричной функции $\gamma \in C^{\infty}\left(S^{1}, U(N)\right)$ к вектор-функции $h \in H=L_{0}^{2}\left(S^{1}, \mathbb{C}^{N}\right)$. Нетрудно проверить (см. книгу [7]), что оператор $M_{\gamma}$ принадлежит унитарной группе $U_{\mathrm{HS}}(H)$, если $\gamma \in C^{\infty}\left(S^{1}, U(N)\right)$.

Вложение $L G \hookrightarrow U_{\mathrm{HS}}(H)$ порождает изометрическое вложение

$$
\Omega G \rightarrow \mathrm{Gr}_{\mathrm{HS}}(H) .
$$

Возвращаясь к задаче о твисторном описании гармонических сфер в пространстве петель $\Omega G$, возникающей в связи с гипотезой о гармонических сферах, заметим, что, пользуясь изометрическим вложением $\Omega G \hookrightarrow \mathrm{Gr}_{\mathrm{HS}}(H)$, можно рассматривать любое гармоническое отображение $\varphi: \mathbb{P}^{1} \rightarrow \Omega G$ как принимающее значение в грассманиане $\mathrm{Gr}_{\mathrm{HS}}(H)$ и, следовательно, в одной из связных компонент $G_{r}(H)$ многообразия $\operatorname{Gr}_{\mathrm{HS}}(H)$. Для описания гармонических отображений $\varphi: \mathbb{P}^{1} \rightarrow G_{r}(H)$ можно воспользоваться теоремой 3 и действовать по аналогии с конечномерной ситуацией.

\section{9. ВОЗВРАЩАЯСЬ К ГИПОТЕЗЕ О ГАРМОНИЧЕСКИХ СФЕРАХ}

Как было указано в разделе 8, мы хотели бы свести доказательство гипотезы о гармонических сферах к голоморфному случаю, "поднимая" обе части соответствия из гипотезы на отвечающие им твисторные пространства.

Сначала хотелось бы получить гармонический аналог теоремы Атьи, утверждающей, что имеется взаимно однозначное соответствие (7). Иначе говоря, хотелось бы получить интерпретацию гармонических сфер в $\Omega G$ в терминах расслоений над $\mathbb{P}^{1} \times \mathbb{P}^{1}$. Попытаемся понять, какой могла бы быть подобная интерпретация.

Предположим, что гармоническая сфера $\varphi: \mathbb{P}^{1} \rightarrow \Omega G \subset \operatorname{Gr}_{\mathrm{HS}}(H)$ является проекцией некоторой гармонической сферы $\widetilde{\varphi}: \mathbb{P}^{1} \rightarrow L G^{\mathbb{C}}$, так что имеет место диаграмма

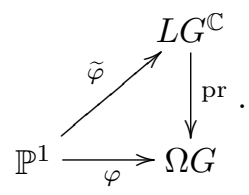

Тогда $\widetilde{\varphi}(z) \in L G^{\mathbb{C}}$ можно рассматривать как функцию перехода некоторого гармонического расслоения над $\mathbb{P}_{z}^{1}$. Это расслоение является ограничением гармонического 
расслоения над $\mathbb{P}^{1} \times \mathbb{P}^{1}$, отвечающего сквозному отображению

$$
\varphi: \mathbb{P}^{1} \ni z \rightarrow \widetilde{\varphi}(z) \in L G^{\mathbb{C}} \rightarrow \varphi(z) \in \Omega G=L G^{\mathbb{C}} / L_{+} G^{\mathbb{C}} .
$$

$\mathrm{B}$ терминах грассманиана $\operatorname{Gr}_{\mathrm{HS}}(H)$ образ $\varphi(z) \in \Omega G \subset \mathrm{Gr}_{\mathrm{HS}}(H)$ должен совпадать с подпространством

$$
W_{z}:=M_{\widetilde{\varphi}(z)} H_{+}
$$

где $M$ - оператор умножения, введенный в конце раздела 8.

Твисторная интерпретация этой конструкции выглядит следующим образом. Гармоническую сферу в $\Omega G$ можно рассматривать как гармоническую сферу в подмногообразии $G_{r}(H) \subset \operatorname{Gr}_{\mathrm{HS}}(H)$, состоящем из подпространств $W \subset H$ некоторой фиксированной виртуальной размерности $r$. В терминах твисторного флагового расслоения гармоническая сфера $\varphi: \mathbb{P}^{1} \rightarrow G_{r}(H)$ является проекцией некоторой $\mathcal{J}_{\sigma}^{2}$-голоморфной сферы $\psi: \mathbb{P}^{1} \rightarrow F_{\mathbf{r}}^{d}(H)$, так что имеется коммутативная диаграмма

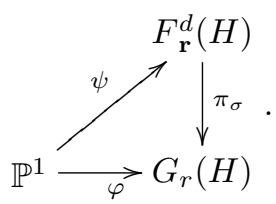

Образ $\psi(z)=\left(\psi_{1}(z), \ldots, \psi_{n}(z)\right)$ точки $z \in \mathbb{P}^{1}$ при отображении $\psi: \mathbb{P}^{1} \rightarrow F_{\mathbf{r}}^{d}(H)$ задается виртуальным флагом $\mathcal{W}_{z}=\left(W_{z}^{1}, \ldots, W_{z}^{n}\right)$. В терминах расслоений над $\mathbb{P}^{1} \times \mathbb{P}^{1}$ это отображение будет допускать следующую интерпретацию. Если каждое отображение $\psi_{i}: \mathbb{P}^{1} \rightarrow G_{r_{i}}(H)$ является проекцией отображения $\widetilde{\psi}_{i}: \mathbb{P}^{1} \rightarrow L G^{\mathbb{C}}$ так, что

$$
W_{z}^{i}=M_{\widetilde{\psi}_{i}} H_{+}
$$

то из описания почти комплексной структуры $\mathcal{J}_{\sigma}^{2}$ на твисторном расслоении $\pi_{\sigma}$ будет следовать, что отображения $\widetilde{\psi}_{i}$ должны задавать либо голоморфное, либо антиголоморфное расслоение над $\mathbb{P}^{1} \times \mathbb{P}^{1}$. По теореме Атьи этим расслоениям должны отвечать либо инстантоны, либо антиинстантоны на $\mathbb{R}^{4}$. Тем самым каждому полю Янга-Миллса на $\mathbb{R}^{4}$ сопоставляется конечный набор инстантонов и антиинстантонов на $\mathbb{R}^{4}$. Эту конструкцию можно рассматривать как твисторную интерпретацию пространства модулей полей Янга-Миллса из гипотезы о гармонических сферах.

Благодарности. При подготовке настоящей статьи автор пользовался частичной поддержкой РФФИ (гранты № 08-01-00014, 09-02-91349), Программы поддержки ведущих научных школ (грант НШ-7675.2010.1) и научной программы Президиума РАН "Нелинейная динамика".

\section{Список литературы}

[1] M. F. Atiyah, Comm. Math. Phys., 93:4 (1984), 437-451.

[2] S. K. Donaldson, Comm. Math. Phys., 93:4 (1984), 453-460.

[3] М. Атья, Геометрия и физика узлов, Мир, М., 1995.

[4] M. F. Atiyah, N. J. Hitchin, I. M. Singer, Proc. R. Soc. Lond. Ser. A, 362:1711 (1978), $425-461$.

[5] J. Eells, S. Salamon, Ann. Scuola Norm. Sup. Pisa Cl. Sci. (4), 12:4 (1985), 589-640.

[6] F. E. Burstall, S. M. Salamon, Math. Ann., 277:2 (1987), 249-265.

[7] Э. Пресли, Г. Сигал, Группы петель, Мир, М., 1990. 